\title{
Neural Control of Motion-to-Force Transitions with the Fingertip
}

\author{
Madhusudhan Venkadesan ${ }^{1,2}$ and Francisco J. Valero-Cuevas ${ }^{2,3,4}$ \\ ${ }^{1}$ Department of Mathematics and ${ }^{2}$ Sibley School of Mechanical and Aerospace Engineering, Cornell University, Ithaca, New York 14853, and ${ }^{3}$ Department \\ of Biomedical Engineering and ${ }^{4}$ Division of Biokinesiology and Physical Therapy, University of Southern California, Los Angeles, California 90089-2905
}

The neural control of tasks such as rapid acquisition of precision pinch remains unknown. Therefore, we investigated the neural control of finger musculature when the index fingertip abruptly transitions from motion to static force production. Nine subjects produced a downward tapping motion followed by vertical fingertip force against a rigid surface. We simultaneously recorded three-dimensional fingertip force, plus the complete muscle coordination pattern using intramuscular electromyograms from all seven index finger muscles. We found that the muscle coordination pattern clearly switched from that for motion to that for isometric force $\sim 65 \mathrm{~ms}$ before contact $(p=0.0004$ ). Mathematical modeling and analysis revealed that the underlying neural control also switched between mutually incompatible strategies in a time-critical manner. Importantly, this abrupt switch in underlying neural control polluted fingertip force vector direction beyond what is explained by muscle activation-contraction dynamics and neuromuscular noise $(p \leq 0.003)$. We further ruled out an impedance control strategy in a separate test showing no systematic change in initial force magnitude for catch trials where the tapping surface was surreptitiously lowered and raised $(p=$ 0.93). We conclude that the nervous system predictively switches between mutually incompatible neural control strategies to bridge the abrupt transition in mechanical constraints between motion and static force. Moreover because the nervous system cannot switch between control strategies instantaneously or exactly, there arise physical limits to the accuracy of force production on contact. The need for such a neurally demanding and time-critical strategy for routine motion-to-force transitions with the fingertip may explain the existence of specialized neural circuits for the human hand.

Key words: motor control; neural control; finger; hand; contact transition; force

\section{Introduction}

Fine manipulation using fingertips is an integral part of the human identity. A fundamental element of manipulation is the acquisition of precision pinch: abruptly making contact with external surfaces to produce static force with the fingertips. Numerous studies show that the apparently trivial task of making contact using any limb is surprisingly difficult to understand (Lacquaniti and Maioli, 1987, 1989; Bizzi et al., 1992; Hogan, 1992; Wolpert et al., 1995; Gribble et al., 1998; Todorov, 2000; Ostry and Feldman, 2003; Kurtzer et al., 2005; Lackner and DiZio, 2005) or to successfully mimic with robots

\footnotetext{
Received May 18, 2007; revised Dec. 7, 2007; accepted Dec. 10, 2007.

This work was supported by the Whitaker Foundation, National Science Foundation (NSF) Grants 0312271 and 0237258, and National Institutes of Health (NIH) Grants HD048566, AR050520, and AR052345 (F.J.V.-C.). M.V. was supported by the NSF Frontiers in Integrative Biological Research Grant 0425878 during manuscript preparation. The contents of this manuscript are solely the responsibility of the authors and do not necessarily represent the official views of the National Institute of Arthritis and Musculoskeletal and Skin Diseases, the National Institute of Childhood and Human Development, the NIH, the Whitaker Foundation, or the NSF. We thank R. V. McNamara III for technical support and experiment execution, F. A. Medina for EMG processing, Dr. V. J. Santos for help with study design, S Song for machining and instrumentation, Drs. M. Price and S. Backus for fine-wire electrodes placement, and Drs. J. Guckenheimer, A. Ruina, M. Srinivasan, K. J. Keenan, E. Todorov, F. Mussa-Ivaldi, and the anonymous reviewers for critical comments.

Correspondence should be addressed to Francisco J. Valero-Cuevas, Department of Biomedical Engineering, University of Southern California, Ronald Tutor Hall 404, 3710 McClintock Avenue, Los Angeles, CA 90089-2905. E-mail: valero@usc.edu.

DOI:10.1523/JNEUROSCI.4993-07.2008

Copyright $\odot 2008$ Society for Neuroscience $\quad 0270-6474 / 08 / 281366-08 \$ 15.00 / 0$
}

(Whitney, 1987; Kazerooni, 1990; Hogan, 1992; Hyde and Cutkosky, 1994; Akella and Cutkosky, 1995; Cavusoglu et al., 1997). Contact discontinuously changes the mechanical constraints of the task. Therefore the joint torques (and muscle forces in turn) for producing motion and force with the endpoint of a limb in a given direction are necessarily different (Hogan, 1985, 1992). However, the underlying neural control strategy need not change. One hypothesis known as impedance control in robotics and equilibrium point control in motor control proposes that to exert a limb force against a surface, we can simply regulate the viscoelastic muscle behavior so as to move the controlled "virtual" position of the limb inside the surface. In this case, a contact force emerges from the mismatch between actual and virtual position, without a direct need to code it in the neural signal (Feldman, 1986; Bizzi et al., 1992; Hogan, 1992; Hyde and Cutkosky, 1994; MussaIvaldi and Bizzi, 2000; Ostry and Feldman, 2003). Alternatively, others hypothesize that the nervous system switches between two distinct control strategies, one to produce limb motion but that cannot produce limb force in the same direction as the motion, and vice versa. Hence, the contact transition is executed through accurate anticipation of contact time and careful feedforward/feedback control of joint torques (Whitney, 1976, 1987; Hogan, 1988; Kazerooni, 1990) or muscle activations (Lacquaniti and Maioli, 1987, 1989; Todorov, 
2000). Regardless of where the reader may stand on this debate, to our knowledge contact transitions have not been studied in the realm of finger function.

Given the biomechanical (Valero-Cuevas et al., 2007) and neural (Penfield and Boldrey, 1937; Schieber and Hibbard, 1993; Scott, 2004) specialization of human fingers, it is unclear whether conclusions from studies of dynamic interactions of whole limbs with the environment extend to neuromuscular control of human fingers. Studies of typing (Kuo et al., 2006) involve tasks where the contact surface is not rigid and the goal is not to produce a well directed sustained static force against it. In contrast, the acquisition of precision pinch for manipulation of rigid objects requires abruptly making contact with the object to immediately generate well directed static fingertip forces. Here, we investigated whether the neural control of finger musculature switches when the index finger abruptly transitions from motion to isometric force production (i.e., a tap-to-push task). When we found that a switch occurred, we studied the timing, mechanical consequences and neuromuscular implications of the switch.

\section{Materials and Methods}

Consenting subjects ( 11 male; 7 female; age range, 19-39 years; mean, 22.8 years) with no history of neurological or hand pathology or injury participated in this study. This study was approved by Cornell University's Committee on Human Subjects. We recorded motions and forces in 18 subjects, and intramuscular electromyograms in nine of them. Subjects wrapped the thumb and unused fingers of their dominant hand around a fixed horizontal dowel to produce force using their index finger against the flat, top surface of a cylindrical pedestal mounted on a six-axis load cell (model 20E12A-I25; JR3, Woodland, CA). The surface position and height was adjusted such that the index finger was in a neutral ad-abduction posture and the metacarpo-phalangeal, proximal-interphalangeal and distalinterphalangeal joints in $\sim 30,45$, and $15^{\circ}$ of flexion, respectively. Resembling our previous work (Valero-Cuevas, 2000), subjects wore a custom-molded thimble with a spherical Teflon bead embedded at its tip. This rigorously defined the mechanical task via a unique contact point and friction cone for force direction.

We instructed subjects to ramp-up fingertip force against a flat target surface to a self-selected high magnitude $[\sim 100 \%$ maximal voluntary contraction (MVC)] as quickly as possible (i.e., tap-topush task). This ensured that the transition from motion to force production was indeed abrupt. They were instructed to, on contact, keep the finger static and the force pointed vertically to the best of their ability. The trials started from three initial conditions: "relaxed," "preactivated," and "motion." In the motion condition, subjects tapped the target surface five times at a $1 \mathrm{~Hz}$ rhythm set by a metronome ( $1 \mathrm{~s}$ for the up-and-down motion) and pushed down on the surface at the end of the fifth cycle (Fig. 1a, blue traces show the last cycle). In the relaxed condition, the subject rested the fingertip on the target surface for two metronome beats (i.e., $2 \mathrm{~s}$ ) before pushing down (Fig. $1 b$, green traces). In the preactivated condition, subjects produced a downward vertical force vector of a self-selected minimal magnitude for two metronome beats before pushing down (Fig. $1 b$, red traces). Subjects typically produced between 0.5 and $1 \mathrm{~N}$ of force magnitude in the preactivated condition.

Electromyograms. The seven muscles actuating the index finger are flexor digitorum profundus (FP), flexor digitorum superficialis (FS), extensor indicis proprius (EI), extensor digitorum communis (EC), first lumbrical (LUM), first dorsal interosseous (DI), and first palmar interosseous (PI). We recorded and digitally processed EMGs using finewire intramuscular electrodes from all seven muscles in nine subjects using previously reported techniques (Valero-Cuevas, 2000). Amplified EMGs were sampled at $2000 \mathrm{~Hz}$, bandpass filtered at $20-800 \mathrm{~Hz}$, full-wave rectified, and normalized by the largest EMG level recorded during maximal voluntary contractions of that muscle. Maximal voluntary contractions of individual muscles were done immediately before and after fingertip force production, with the index finger braced in the same posture used during the study. We multiplied the normalized EMG from each muscle by its maximal muscle stress and then by its physiological cross-sectional area values obtained by us in three previous biomechanical studies (Valero-Cuevas et al., 1998; Valero-Cuevas, 2000; Valero-Cuevas and Hentz, 2002) to find a timevarying muscle-force vector $[\mathbf{m}(t)$, the "muscle coordination pattern" (Valero-Cuevas et al., 1998; Valero-Cuevas, 2000, 2005)] [FP, FS, EI, EC, LUM, DI, PI $]^{T}$. Finally, we smoothed $\mathbf{m}(t)$ using a symmetric moving average with a $50 \mathrm{~ms}$ window. As in our past work (ValeroCuevas, 2000), the reference coordination pattern vector $\left(\mathbf{m}^{\text {ref }}\right)$ for each trial was defined as the average of $\mathbf{m}(t)$ during $100 \mathrm{~ms}$ of peak force (always occurring $>500 \mathrm{~ms}$ after contact). We calculated the angle $[\theta(t)]$ between $\mathbf{m}(t)$ at every sample and $\mathbf{m}^{\text {ref }}$ using the unitvector dot-product formula given as follows:

$$
\theta(t)=\cos ^{-1}\left(\frac{\mathbf{m}(t) \cdot \mathbf{m}^{r e f}}{\|\mathbf{m}(t)\|\left\|\mathbf{m}^{r e f}\right\|}\right)
$$

where a larger $\theta$ means a larger misalignment of the measured coordination pattern with respect to the reference pattern, and $\theta=0$ means perfect alignment.

We found average of $\theta$ for each trial in six windows of 50 ms width as shown in Figure $2 a$, performed one-way repeated measures ANOVA to test whether $\theta$ differed across the six time intervals. We applied a Tukey-Kramer adjustment for multiple comparisons when reporting $p$ values. We verified that the residuals were normally and identically distributed.

Fingertip force analysis. We used the vertical force $\left(F_{\mathrm{z}}\right)$ to detect contact or start of force-ramps. Force production was said to start when $F_{z}$ exceeded 6 SDs above mean $F_{z}$ during the relaxed, preactivated or nocontact period. Through trial and error we found a threshold of 6 SDs to yield the most reliable detection of force onset and manually verified each automatically detected force onset. We discarded trials where the finger slipped or bounced after contact, or if the flight phase during motion trials was $<400 \mathrm{~ms}$ (despite the metronome).

We low-pass filtered ( $80 \mathrm{~Hz}$ cutoff) the force data from all three axes before calculating the angular deviation of the force vector from vertical $\left[\phi_{\text {force }}(t)\right]$. We then found $\max \left(\phi_{\text {force }}\right)$ and $\operatorname{var}\left(\phi_{\text {force }}\right)$ in the time interval between +10 and $+65 \mathrm{~ms}$ after contact (see Fig. $3 a-c$ ). To be judiciously conservative, we excluded force data for the first 10 ms after contact to remove high-frequency sensor noise transients that subdued typically by $5 \mathrm{~ms}$, and always well within $10 \mathrm{~ms}$ (Fig. 1a). We also limited our analysis to +65 ms because sensory feedback will affect force production subsequently (Venkadesan et al., 2007). Both $\max \left(\phi_{\text {force }}\right)$ and $\operatorname{var}\left(\phi_{\text {force }}\right)$ were log transformed to ensure normality. We then performed repeated measures ANOVA to test for differences in $\max \left(\phi_{\text {force }}\right)$ and $\operatorname{var}\left(\phi_{\text {force }}\right)$ between different initial conditions. We applied a Tukey-Kramer adjustment for multiple comparisons when reporting $p$ values.

Index finger model. To objectively evaluate the mechanical consequences of the contact transition on the initial angular deviation of the force vector, we developed a torque-driven planar index finger model with an ideal inelastic contact collision. This model produced motion when unconstrained, or force immediately on contact with a surface (for nonslip conditions). Finger motion was modeled using a three-link open kinematic chain; and initial force production as a four-bar linkage closed kinematic chain. We switched between the separate formulations for the motion and force production at contact.

We calculated net joint torques for motion $\left(\boldsymbol{\tau}_{\text {motion }}\right)$ while ensuring that two features of our experimental trials were emulated. First, we designed model finger kinematics to emulate the subjects' preference to keep the distal phalanx vertical (i.e., perpendicular to the target surface). Second, to emulate the fact that subjects' fingertip made contact with a nonzero vertical velocity, we prescribed a sigmoidal vertical velocity profile for the model fingertip, starting with zero velocity $500 \mathrm{~ms}$ before contact (highest finger posture) and peak velocity at contact. These two 

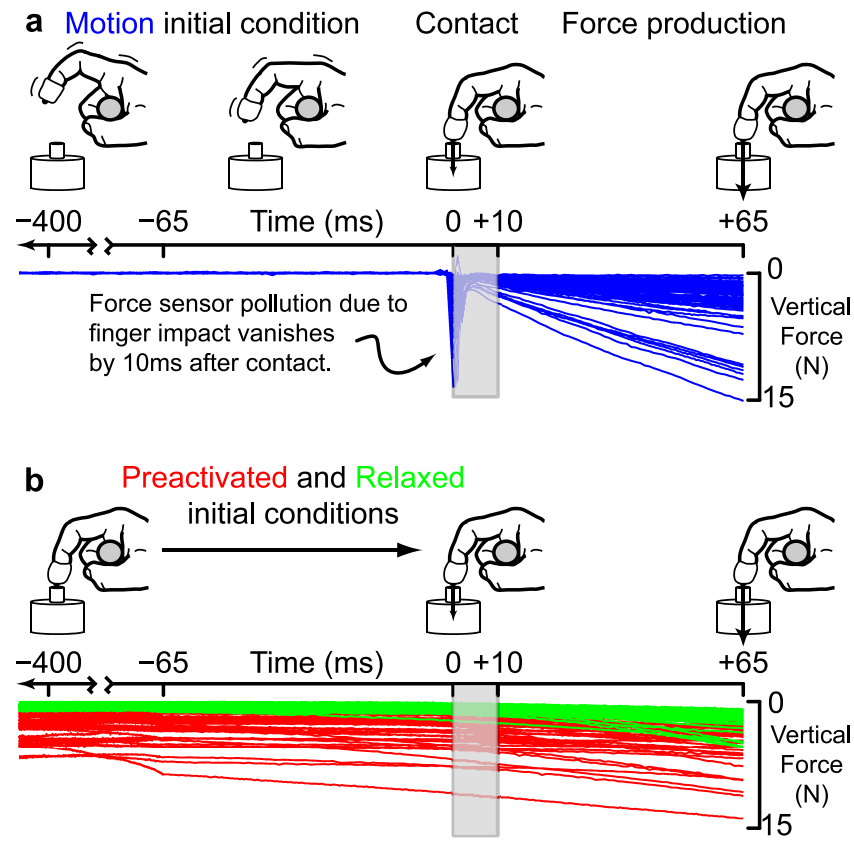

Figure 1. Experimental setup and force production under different initial conditions. $\boldsymbol{a}$, For the motion initial condition, subjects tapped the surface five times before pushing against it to maximize vertical force. The blue traces show vertical force data from all subjects. Data from the first $10 \mathrm{~ms}$ (gray box) were excluded to remove high-frequency impact sensor noise transients that subdued typically by $5 \mathrm{~ms}$, and always well within $10 \mathrm{~ms}$. $\boldsymbol{b}$, For the relaxed (green) and preactivated (red) initial conditions, subjects produced force-ramps in a static posture (no previous finger motion), and from nearly zero, or low force ( $\sim 25 \%$ of maximal voluntary force), respectively.

requirements uniquely specified the joint angles $[\boldsymbol{\varphi}(t)]$ and joint angular velocities/accelerations throughout the motion phase. Given that the left-hand side of Equation 2 is known for every instant of time during the motion phase, we directly calculated the unique joint torques for producing this motion:

$$
M(\boldsymbol{\varphi}) \ddot{\boldsymbol{\varphi}}+C(\boldsymbol{\varphi}, \dot{\boldsymbol{\varphi}}) \dot{\boldsymbol{\varphi}}+\mathrm{N}(\boldsymbol{\varphi})=\boldsymbol{\tau}_{\text {motion }}
$$

where $\varphi$ is the vector of joint angles, $M$ represents the inertial properties of the finger, $C$ represents Coriolis and centrifugal forces on each phalanx, and $\mathbf{N}$ is the gravitational term.

The dynamical equation for initial force production when the fingertip makes contact with the surface is as follows:

$$
M(\boldsymbol{\varphi}) \ddot{\boldsymbol{\varphi}}+C(\boldsymbol{\varphi}, \dot{\boldsymbol{\varphi}}) \dot{\boldsymbol{\varphi}}+\mathrm{N}(\boldsymbol{\varphi})+A(\boldsymbol{\varphi})^{T} \mathbf{f}=\boldsymbol{\tau}_{\text {force }},
$$

where

$$
\mathbf{f}=\left(A M^{-1} A^{T}\right)^{-1}\left(A M^{-1}\left(\boldsymbol{\tau}_{\text {force }}-C \dot{\boldsymbol{\varphi}}-N\right)+\dot{A} \dot{\boldsymbol{\varphi}},\right.
$$

where the posture dependent matrix $A$ is the manipulator Jacobian that maps joint angular velocities to translational velocities of the fingertip and $\mathbf{f}$ is the fingertip force vector produced when in contact with a surface. The beaded thimble used in the experiment precludes the fingertip from producing any torques while maintaining a static posture (for a detailed description of the torque output of the fingertip, see Valero-Cuevas et al., 1998). By definition, the unique joint torques needed to produce static vertical force $\left(\boldsymbol{\tau}_{\text {force }}\right)$ are calculated by setting joint angular velocities and accelerations to zero in Equations 3 and 4 ; in other words, $\boldsymbol{\tau}_{\text {force }}=A(\varphi)^{T} \mathbf{f}+\mathbf{N}(\varphi)$, where $\mathbf{f}$ is the desired fingertip force vector.

We calculated $\tau_{\text {motion }}(t)$, such that under the assumptions outlined for Equation 2, it would take $500 \mathrm{~ms}$ to travel from the height of the metacarpo-phalangeal joint to the height of the target. Also, the posture at contact emulated the reference posture used in our experiments, namely, 30,45 , and $15^{\circ}$ of flexion, from the proximal to the distal joints, respectively. We calculated joint torques at contact such that for the reference contact posture, $\boldsymbol{\tau}_{\text {force }}$ would produce static vertically oriented fingertip force vector of $0.15 \mathrm{~N}$ in magnitude. We simulated various durations ( 0 to $65 \mathrm{~ms}$ ) of transition between $\boldsymbol{\tau}_{\text {motion }}$ and $\boldsymbol{\tau}_{\text {force }}$, as well as various errors in timing, i.e., the instant when the transition is completed ( 0 to $20 \mathrm{~ms}$ before contact occurs). We assumed an ideal inelastic collision (i.e., fingertip and joint motions come to a standstill on contact). This allowed us to examine the mechanical consequences (i.e., causal relationships) between a noninstantaneous transition in joint torques and initial misdirection in fingertip force vector on contact, independently of the viscoelastic properties of the finger and collision dynamics.

Test of impedance control. We directly tested whether impedance control was used in a parallel study with six consenting subjects (three male; three female; average age, 19.5 years; range, 19-20 years) in which EMGs were not recorded. The task was identical to the with motion condition, with the difference that blindfolded subjects, while wearing the thimble, continuously tapped a $50 \mathrm{~mm}$ diameter rough sensor surface. Each tap motion lasted 3 s: the up-down tapping motion lasted $1 \mathrm{~s}$ as in the "motion" initial condition, followed by a $2 \mathrm{~s}$ period when subjects pushed to a self-selected low force $(\sim 25 \%$ MVC). Each subject performed a total of 150 taps in 10 batches of 15 taps. For all taps a robot lowered the surface to touch a bell, to allow the subject to phase-lock their tapping cadence, and then randomly raised the surface to either the reference height (in $95 \%$ of the taps) or to a different height (in 5\% of the taps). The surface height for the $5 \%$ of "catch" trials was selected at random from a uniform distribution with $\pm 6 \mathrm{~mm}$ range. This prevented the blindfolded subject from using auditory cues to identify catch trials. The dependent variable for the regression (Fig. 5) was peak $\|\boldsymbol{F}\|$ for $t=+10 \mathrm{~ms}$ to $t=+65 \mathrm{~ms}$ after contact, normalized by the steady-state force the subjects reached at each tap. The variability in steady state force across subjects precluded our detecting systematic changes (if any) in initial force magnitude with surface height. Thus, we used normalization as a means to best detect a systematic change in initial force magnitude across trials with disparate self-selected steady-state static force magnitude.

\section{Results}

The 7D muscle coordination patterns $[\mathbf{m}(t)]$ for motion (Fig. $2 a$, dark boxes) and force production (Fig. $2 a$, light boxes) were significantly different $\left(\Delta \theta \geq 20^{\circ}, p<0.0001\right)$. However, even $65 \mathrm{~ms}$ before contact (Fig. $2 a$, during finger motion, shaded box), the coordination pattern had already changed abruptly (in a span of $\sim 60 \mathrm{~ms}$ ) and was significantly different from the preceding periods of finger motion (Fig. $2 a$, dark box at $[-150,-100]$ vs shaded box at $[-90,-40])\left(\Delta \theta=14^{\circ}, p=\right.$ $0.0004)$, but statistically indistinguishable from the pattern for static force production (Fig. $2 a$, light box at $[+20,+70]$ vs shaded box at $[-90,-40])\left(\Delta \theta=6^{\circ}, p=0.28\right)$. This clear and abrupt transition between coordination patterns occurred before contact could alter the mechanical condition (i.e., the switch in muscle coordination pattern cannot be explained by the onset of contact). Moreover, the coordination pattern $[\mathbf{m}(t)]$ after contact was well aligned with $\mathbf{m}^{\text {ref }}$, the reference pattern for maximal static force production (Fig. $2 a$, light boxes) $\left(\theta<20^{\circ}\right)$.

In addition, the increase in the vector magnitude of the muscle coordination pattern parallels that for the vector direction but is initiated later (i.e., closer to the contact time) (Fig. 2b). That is, the nervous system begins by increasing the alignment of the coordination pattern vector with that for force production over $100 \mathrm{~ms}$ before contact (Fig. 2a). In contrast, the vector magnitude of the coordination pattern begins to increase to match that for initial force production $<70$ ms before contact (Fig. 2b). Thus, we find that the transformation of the muscle coordination pattern vector from that 


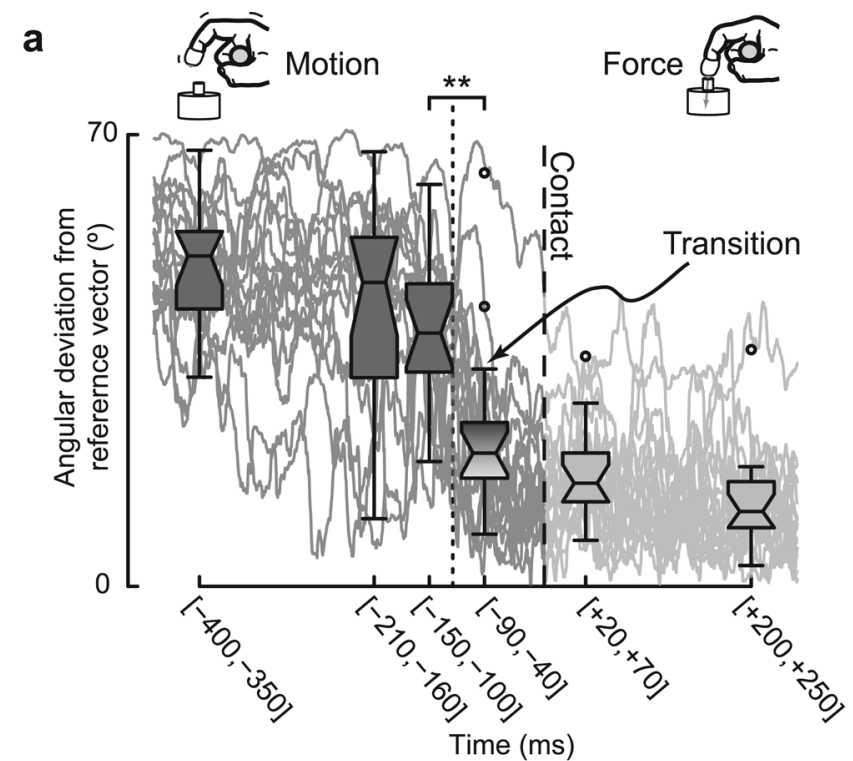

b

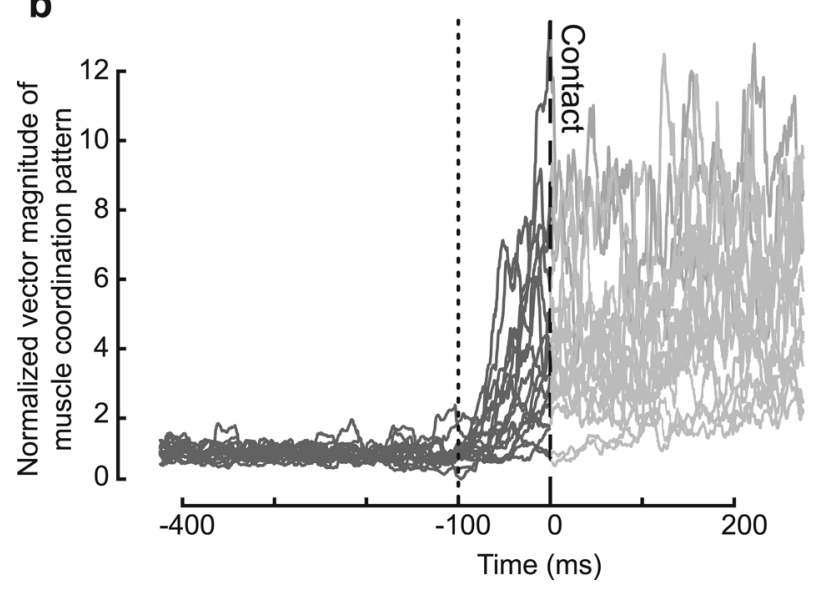

Figure 2. Switch in direction and magnitude of the muscle coordination pattern vector between motion and static force production. $\boldsymbol{a}$, Muscle coordination patterns (dark and light traces) are represented by their angular deviation $(\theta)$ from a reference coordination pattern (namely, the coordination pattern when fingertip force was the highest). The box plots are 50 ms wide averages of $\theta$. The central line is the median, the notches are the robust $95 \%$ confidence limits of the median, the lower and upper limits of the box are the quartile limits, whisker lengths are 1.5 times the interquartile distance, and outliers are shown with circular markers. The switch between patently different patterns for motion and force occurred very abruptly and before contact (the shaded box extending from $-90 \mathrm{~ms}$ to $-40 \mathrm{~ms}$ ). $\boldsymbol{b}$, The vector magnitude of the muscle coordination pattern was normalized by the mean magnitude for each trial during the first $200 \mathrm{~ms}$ of motion. This enabled an objective comparison of how the magnitude scaled over the time course of each trial. The vector magnitude was higher even for initial force production compared with the motion phase. Much like the switch in vector direction $(\boldsymbol{a})$, this increase in vector magnitude started before contact. However, unlike the vector direction that started switching over $100 \mathrm{~ms}$ before contact, the vector magnitude started increasing less $\sim 70$ ms before contact.

for motion to that for static force resembles a nonlinear interpolation.

A mathematical analysis of the time course of the transition of muscle coordination patterns shows that there was necessarily a switch between mutually incompatible underlying neural control strategies: from one for finger motion to another for fingertip force production (for full details, see the supplemental material, available at www.jneurosci.org). By "incompatible" neural control strategies we mean incompatible with each other by virtue of each strategy being able to meet only one set of mechanical constraints: either those for motion or those for force. Briefly, our analysis formalizes the following statement: given that the finger's mechanical state and external constraints do not change as it approaches contact, a change in muscle coordination pattern necessitates a change in the underlying neural control strategy. That is, the switch in muscle coordination pattern is of neural origin because it cannot be explained by the mechanical transition at contact. The proof of this argument follows the reductio ad absurdum argument. If one assumes the underlying neural control strategy to be constant as the finger undergoes contact, then the resulting relationship among muscle coordination patterns directly contradicts our experimental finding that they change rapidly before contact. This result applies even when the underlying neural control strategy undergoes changes compatible with neural and muscle redundancy (e.g., changes in neural signals that do not affect the muscle coordination patterns). Importantly, this analytical conclusion holds for a general underlying neural control strategy that could, for example, encode either muscle forces directly, or other properties of the neuromuscular system like motoneuron excitability.

We also found that the abrupt switch in underlying neural control strategy polluted fingertip force vector direction beyond what is explained by muscle activation-contraction dynamics (Zajac, 1989), neuromuscular noise (Harris and Wolpert, 1998), and premotor planning (Sober and Sabes, 2005). We used two quantifiers of fluctuations in force production between +10 and +65 ms after contact (Fig. 3 ): peak angular deviation $\left[\phi_{\text {force }}(t)\right]$ of the fingertip force vector from the vertical $\left[\max \left(\phi_{\text {force }}\right)\right]$ and its variance $\left[\operatorname{var}\left(\phi_{\text {force }}\right)\right]$. Repeated measured ANOVA found both $\max \left(\phi_{\text {force }}\right)$ and $\operatorname{var}\left(\phi_{\text {force }}\right)$ to be significantly larger for the motion initial condition, and significantly and progressively lower for the "relaxed" and "preactivated" initial conditions (Fig. 3; highest post hoc pairwise $p$ value $<0.018$ ).

Importantly, our mechanical torque-driven planar index finger model found that switching between control strategies is time critical, and the likely source of pollution in force direction. Such force misdirection on contact is predicted to occur in this model even though the contact collision is ideally inelastic and the viscoelastic properties of the finger and force transducer are not present. If the switch in neural control strategy could occur instantaneously and exactly on contact (i.e., resembling an ideal step function at contact) the model predicted the fingertip force vector to be perfectly perpendicular to the surface immediately after contact (Fig. 4, origin). However, a more realistic sample simulation compatible with muscle excitation-contraction delays where the transition takes $35 \mathrm{~ms}$ to be completed and ends exactly at contact shows that the fingertip force misdirection at the instant of contact is at least $7^{\circ}$ (Fig. 4), which is realistically compatible with our experimental results. Moreover, repeated iterations of the model show that the force vector misdirection is very sensitive to both the timing and duration of the transition. Force misdirection errors arise simply because transition duration and/or imprecise timing cause the finger to make contact in a posture different from the planned posture. Figure 4 underscores the time criticality of this switching in control strategies because even small (i.e., $10 \mathrm{~ms}$ ) increases in both the onset and duration of the transition can lead to $>60 \%$ increase of errors in initial force vector direction (Fig. 4) (e.g., vertical line at 35 
ms). The effectiveness of compensatory strategies in the face of such time criticality is addressed in the Discussion.

Last, a necessary prediction of impedance control is that force output $(\|\boldsymbol{F}\|$ shortly after contact) will systematically change with surface height. However, we saw no such systematic change in the catch trials where subjects tapped on the surreptitiously elevated/lowered surface $(n=6$; $p=0.93)$ (Fig. 5).

\section{Discussion}

By recording EMGs from all muscles of the index finger, we found that muscle coordination patterns changed abruptly in a nonlinear, time-critical manner between characteristic motion and "force" patterns. Because this clear and abrupt transition occurred before contact could alter the finger's mechanical condition, our mathematical analysis finds this necessarily reflects a switch between mutually incompatible underlying neural control strategies. Additionally, because force output did not vary systematically with surreptitious changes in the tapping surface height, we could directly rule out impedance control as the dominant form of control. Importantly, we find that this switch between underlying neural control strategies results in pollution of the initial fingertip force vector direction above and beyond neuromuscular noise or muscle activation-contraction dynamics. A mechanical index finger model found that these unavoidable errors in initial force direction are a consequence of the inability of the nervous system to switch instantaneously and exactly between incompatible control strategies. Thus, the physiological limitations of the neuromuscular system plus the time criticality of this switching in control strategies conspire to impose physical limits on the accuracy of force production on contact. We speculate that such a neurally demanding and timecritical strategy for the transition from the control of fingertip motion to force production may explain the existence of specialized neural circuits for the human hand.

Finding a switch between mutually incompatible underlying neural control strategies has important consequences to the study of motor control of the fingers. For human fingers, our findings challenge hypotheses like equilibrium point control that propose constant control strategies capable of bridging abrupt changes in mechanical constraints (Feldman, 1986; Bizzi et al., 1992; Gribble et al., 1998; Mussa-Ivaldi and Bizzi, 2000; Ostry and Feldman, 2003). A proposed advantage of equilibrium
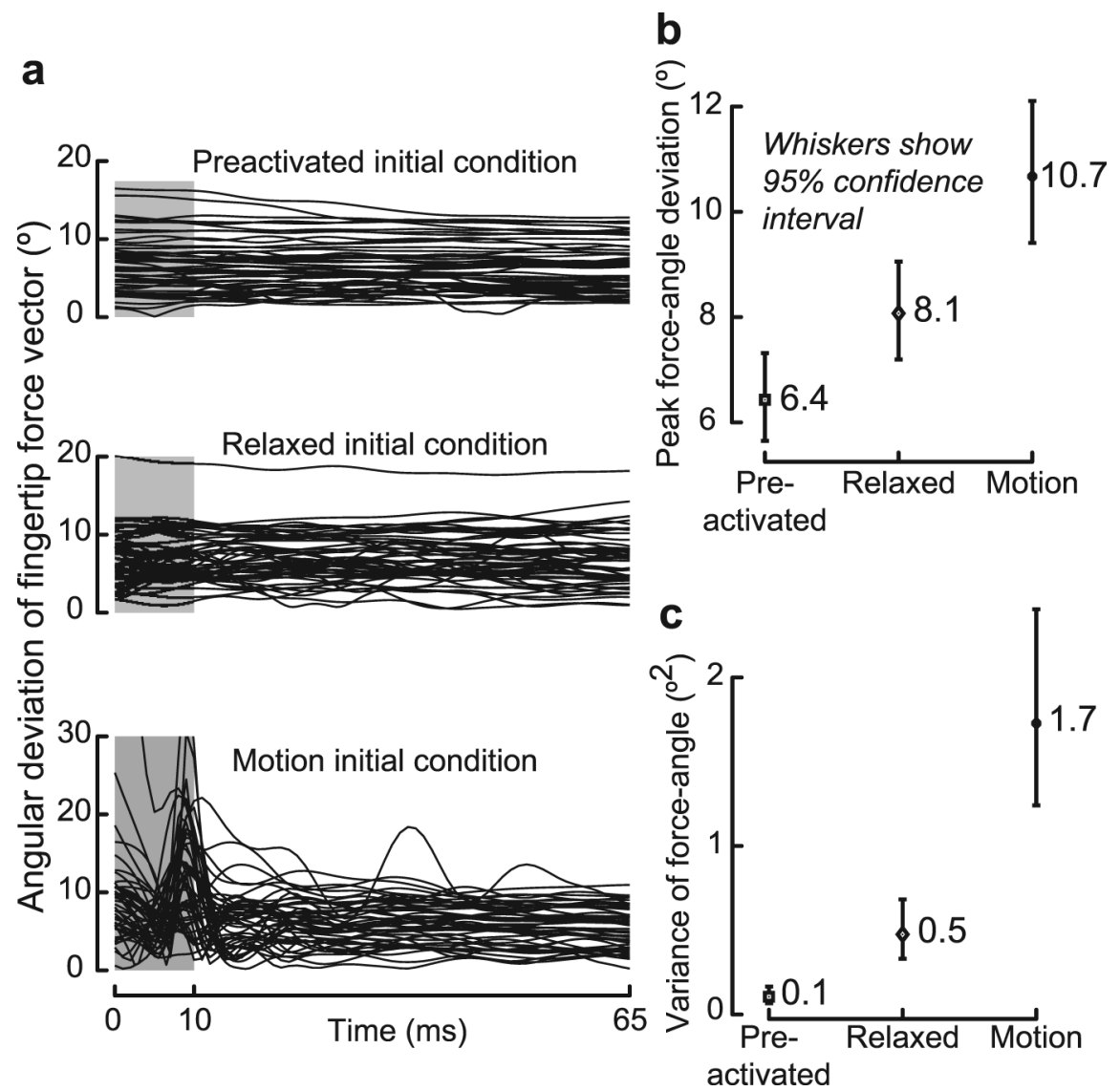

Figure 3. Fluctuations in direction of the fingertip force vector after finger contact. $\boldsymbol{a}$, Force angle data $\left[\phi_{\text {force }}(t)\right]$ from all subjects for all three initial conditions. The first $10 \mathrm{~ms}$ are grayed out and excluded for all analyses. Even for the remaining time segment, the force direction appears to fluctuate much more for the motion condition than for either the relaxed or preactivated conditions. The peak force angle deviation for the motion condition (blue) occurs around $8 \mathrm{~ms}$. We are conservative in our analyses by excluding the first $10 \mathrm{~ms}$ because the force data were clearly reliable well before $8 \mathrm{~ms}$ (Fig. 1a, blue traces). $\boldsymbol{b}$, The peak force angle deviation $\left[\max \left(\phi_{\text {force }}\right)\right]$ decreased from motion to relaxed to preactivated conditions. All differences were statistically significant. $c$, The variance in force direction $\left[\operatorname{var}\left(\phi_{\text {force }}\right)\right]$ also decreased from motion to relaxed to preactivated conditions and all differences were statistically significant.

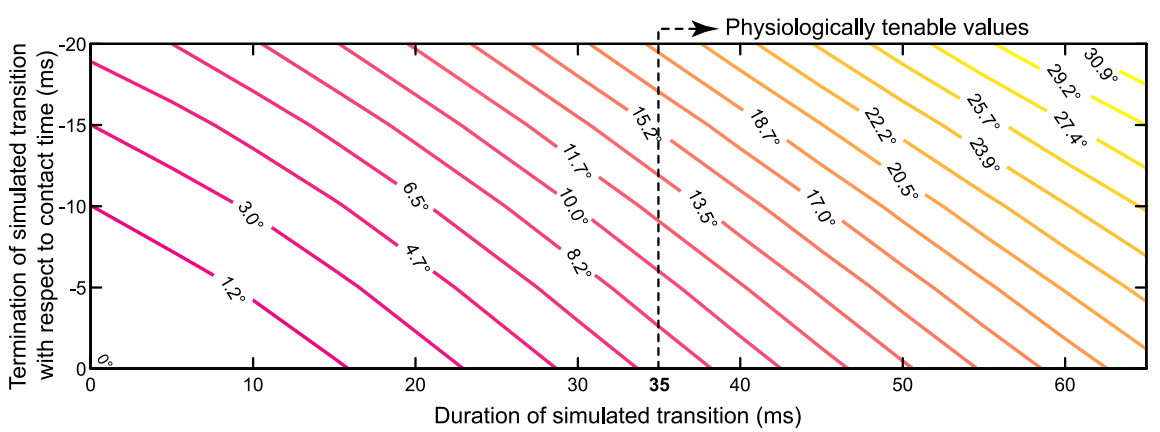

Figure 4. Simulations reveal that just muscle activation-contraction dynamics impose a physical limit on directional accuracy of the initial fingertip force vector on contact. The labeled contour lines show the misdirection of the initial fingertip force vector with respect to the surface normal. The abscissa shows the duration of the transition between the joint torque pattern for motion and that for force; and the ordinate shows inaccuracies in the timing of this transition, represented by its termination time with respect to contact time. For physiologically tenable values of transition duration ( $>35 \mathrm{~ms}$ ), the initial fingertip force vector is misdirected by at least $7^{\circ}$, and the misdirection increases by over $60 \%$ for every $10 \mathrm{~ms}$ increase in timing inaccuracy (contour values along vertical dotted line at $35 \mathrm{~ms}$ abscissa).

point control is that it can mediate transitions between posture and movement "automatically," without the nervous system explicitly computing or actively controlling it (Ostry and Feldman, 2003). In contrast, our data and analysis show a clear example of 


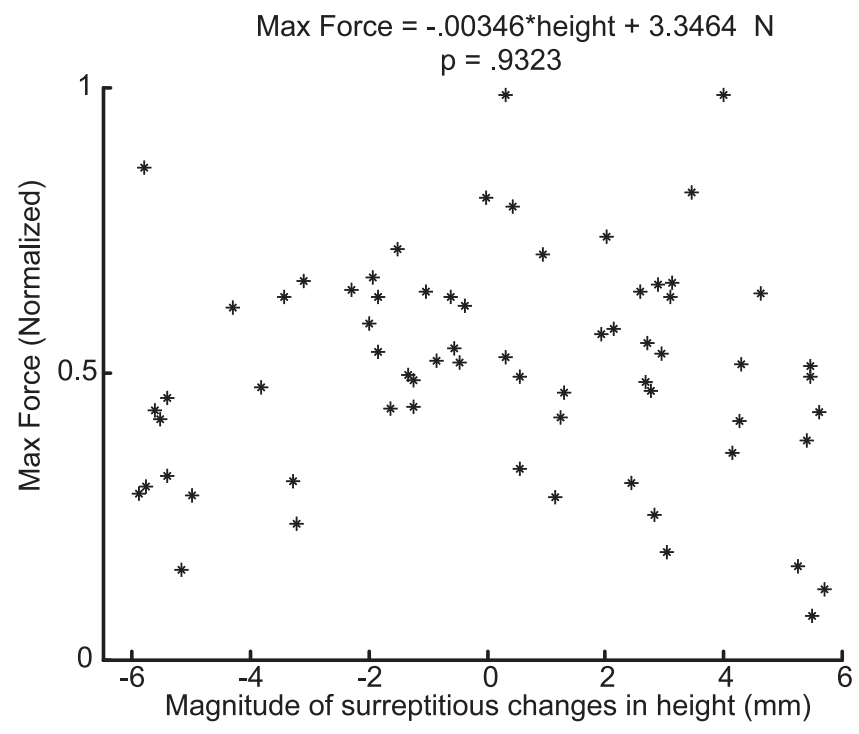

Figure 5. Simple impedance control was not observed. The peak fingertip force magnitude (ordinate) within $65 \mathrm{~ms}$ after contact (the first $10 \mathrm{~ms}$ excluded) did not change systematically with surreptitious changes in the surface height (abscissa). This shows that a simple impedance control strategy could not have been the dominant form of control for finger contact transitions.

the nervous system using a neurally demanding and time-critical switch between mutually incompatible underlying neural control strategies to transition from motion to force production. Independently of current debates on plausible control strategies, our results support the more fundamental idea that the neural control of the fingers fits well within the emerging framework of hybrid control systems characterized by dynamical systems subject to continuous controls and discrete transitions (Guckenheimer, 1995; Branicky et al., 1998).

Our results reveal that the time-critical transformation of the muscle coordination pattern vector before contact resembles a nonlinear transformation. We find that the change in the vector direction of the muscle coordination pattern is well underway at least $100 \mathrm{~ms}$ before contact (Fig. $2 a$ ). In contrast, the vector magnitude begins to change only within $70 \mathrm{~ms}$ of contact (Fig. 2b). A linear transformation would cause simultaneous and proportional scaling of both the vector magnitude and direction. Three possible explanations come to mind: (1) there exist neuro-musculo-skeletal constraints which prevent or bias against the implementation of a linear transformation (e.g., neural coupling, motoneuron pool spillover, anatomical coupling among finger musculature); (2) the limitations of EMG artifactually distort estimates of linearity of the transformation; or (3) a nonlinear path is conducive to reducing errors in the fingertip trajectory, the finger posture at contact and, hence, initial force production. We have previously presented fine-wire EMG evidence that the nervous system is able to control index finger muscles independently in a similar force production paradigm (Valero-Cuevas et al., 1998; Valero-Cuevas, 2000). This argues against neuromusculo-skeletal constraints or EMG artifacts being the dominant explanation for the nonlinear transformation in our present data. By this process of elimination we speculate that, while the finger is still in motion, the nervous system reduces the deviation from the planned posture for contact by postponing the necessary increase in coordination pattern magnitude. Establishing whether this specific nonlinear transformation is task-optimal requires additional experimental and modeling work that is beyond the scope of this first report of the phenomenon. The time criticality of this transformation is discussed below.

Our work extends current understanding of the control of finger musculature by showing that during abrupt transitions between neural control strategies, muscle physiology imposes physical limits to the accuracy of static force production on contact. Our simulations quantify the mechanical sensitivity of initial force direction to the duration and timing of the switch between mutually incompatible strategies (Fig. 4). That is, switching between mutually incompatible control strategies for motion and static force will unavoidably pollute force production after contact if not done exactly and instantaneously. For realistic switching durations constrained at a minimum by $35 \mathrm{~ms}$ of muscle activation-contraction dynamics (Zajac, 1989), deviations from a planned completion of the switching by even $10 \mathrm{~ms}$ increase force misdirection by $>60 \%$ (Fig. 4, vertical line at $35 \mathrm{~ms}$ in duration). In the biological system, timing errors of $\sim 10 \mathrm{~ms}$ are tenable given unavoidable neuromuscular noise (Harris and Wolpert, 1998) and physiological delays (Venkadesan et al., 2007). It is conceivable that the nervous system could plan joint torques for motion using compensatory strategies so that the finger lands in the planned contact posture. However, any such anticipatory motor planning does not, in practice, suffice to cancel out errors in initial force direction because it necessarily involves at the very least an accurate estimate of the time of contact with the surface. As mentioned above, even a $10 \mathrm{~ms}$ uncertainty in the estimate of contact time suffices to incur substantial errors in initial force direction of the magnitudes we saw experimentally. This may explain why our healthy and motivated subjects always exhibited a misdirected initial force. After this initial misdirection, musculoskeletal viscoelastic properties and sensory feedback control will undoubtedly take effect in the real finger and cause the observed force oscillations and subsequent refinement of force direction (Fig. 3).

Our experimental design allowed the disambiguation between mechanical, muscular and neural sources of inaccuracies of force production. Comparing across the motion, "preactivated" and "relaxed" initial conditions allowed us to directly identify fluctuations in force direction for the first $65 \mathrm{~ms}$ after onset as arising from switching between neural control strategies, as distinct from the effects of premotor planning and muscle activationcontraction dynamics. As previously shown by Valero-Cuevas (2000), the preactivated condition likely only requires scaling of the coordination pattern and is therefore only affected by neuromuscular noise. The relaxed condition, however, is additionally affected by the selection and implementation of the motor program (e.g., premotor planning plus muscle activationcontraction dynamics).

We now discuss the relationships of our findings to past work on anticipatory motor control, and the limitations of our approach. Multiple studies have characterized anticipatory control in the limbs of humans and animals (1) for smooth motion-force tasks for limbs subject to contacting [e.g., manipulanda experiments (Shadmehr and Mussa-Ivaldi, 1994; Lackner and DiZio, 2005)] and noncontacting [e.g., Coriolis force experiments (Lackner and DiZio, 2005)] force fields, (2) during abrupt postural perturbations associated with catching (Lacquaniti and Maioli, 1989; Lacquaniti et al., 1992), and (3) and animal studies of posture vs ground-reaction force control (Lacquaniti and Maioli, 1994) or posture versus movement control (Kurtzer et al., 2005). Our results agree with 
their findings and conclusions to the extent that the nervous system can and does effectively anticipate changes in task constraints. In addition, the timing of the onset of anticipatory changes in EMGs of $\sim 100 \mathrm{~ms}$ are similar to those found previously (Lacquaniti and Maioli, 1989). However, anticipatory control in our task is different from braking to mitigate a collision or from stiffening before catching. We did not observe any anticipatory braking because the goal of our task required a collision so as to be able to ramp up force production as rapidly as possible, as evinced by the spikes in vertical force at contact (Fig. 1a). To our knowledge, this is the first study to record complete muscle coordination patterns and full three-dimensional force vectors while fingertips abruptly contact a surface to produce static force. This enabled us to use careful mathematical analysis and mechanical simulations to (1) uncover the nonlinear nature of the anticipatory transformation of muscle coordination patterns, (2) detect the switch in the underlying neural control strategy, and (3) characterize the consequences of this switch to the accuracy of initial force production. Unlike previous reports, our conclusions are independent of any one specific theory of motor control (e.g., equilibrium point hypothesis, direct cortical control of muscles, etc.). We believe the limitations of our approach do not challenge the validity of our conclusions. Although the use of a custom-molded thimble may appear unnatural, its potential drawbacks are outweighed by the benefits of a well defined contact condition (e.g., fingernail length and shape, skin dryness). This has allowed us to obtain high-fidelity biomechanical recordings that can be well approximated by a model (Valero-Cuevas et al., 1998; Valero-Cuevas, 2000). In addition, the use of the thimble does approximate precision pinch acquisition of small, irregularly shaped or slippery objects. Other considerations point us to future studies such as the effect of lengthy practice on the neural strategy, or the inclusion of specialized populations such as microsurgeons or pianists. Similarly, the torque-driven model of the index finger can be further explored through parameter sensitivity analysis (Santos and Valero-Cuevas, 2006) or extended to include musculature, sophisticated control strategies, and contact collision models.

In conclusion, contacting a surface with the fingertip to produce static force requires (1) accurate prediction of when contact would occur and (2) time-critical switching between underlying neural control strategies. For these reasons, dedicated neural circuits are likely used for representation of motion and force similar to the observation for control of the arm in monkeys (Kurtzer et al., 2005). Interestingly, it is known that in humans direct corticospinal projections to the hand muscles are more prevalent than for the limbs (Scott, 2004). Therefore our results could provide a functional insight into an important evolutionary feature of the human brain: the disproportionately large sensory and motor representations of the hand (Penfield and Boldrey, 1937). If indeed the nervous system faced evolutionary pressures to precisely and anticipatorily control even routine tasks like rapid acquisition of precision pinch, the sensorimotor cortical representations of our fingers would naturally reflect those requirements for careful timing of motor actions and fine independent control of the finger muscles. Finally, our finding of the stringent sensorimotor demands of finger contact transitions might also help understand why precision pinch and finger tapping are skills that take years to develop in young children (Forssberg et al., 1991) and are so vulnerable to neurological degeneration and aging (Cole et al., 1998).

\section{References}

Akella PN, Cutkosky MR (1995) Contact transition control with semiactive soft fingertips. IEEE Trans Rob Autom 11:859-867.

Bizzi E, Hogan N, Mussaivaldi FA, Giszter S (1992) Does the nervoussystem use equilibrium-point control to guide single and multiple joint movements. Behav Brain Sci 15:603-613.

Branicky MS, Borkar VS, Mitter SK (1998) A unified framework for hybrid control: model and optimal control theory. IEEE Trans Automat Contr 43:31-45.

Cavusoglu M, Yan J, Sastry S (1997) A hybrid system approach to contact stability and force control in robotic manipulators. In: Proceedings of the 1997 IEEE International Symposium on Intelligent Control, pp 143-148. Piscataway, NJ: IEEE.

Cole KJ, Rotella DL, Harper JG (1998) Tactile impairments cannot explain the effect of age on a grasp and lift task. Exp Brain Res 121:263-269.

Feldman A (1986) Once more on the equilibrium-point hypothesis (lambda model) for motor control. J Mot Behav 18:17-54.

Forssberg H, Eliasson A, Kinoshita H, Johansson R, Westling G (1991) Development of human precision grip I: Basic coordination of force. Exp Brain Res 85:451-457.

Gribble PL, Ostry DJ, Sanguineti V, Laboissiere R (1998) Are complex control signals required for human arm movement? J Neurophysiol 79:1409-1424.

Guckenheimer J (1995) A robust hybrid stabilization strategy for equilibria. IEEE Trans Automat Contr 40:321.

Harris CM, Wolpert DM (1998) Signal-dependent noise determines motor planning. Nature 394:780-784.

Hogan N (1985) The mechanics of multi-joint posture and movement control. Biol Cybern 52:315-331.

Hogan N (1988) On the stability of manipulators performing contact tasks. IEEE J Rob Autom 4:677-686.

Hogan N (1992) Control of contact in robots and biological systems. IEEE Eng Med Biol Mag 11:81-82.

Hyde J, Cutkosky M (1994) Controlling contact transition. IEEE Contr Systems Mag 14:25-30.

Kazerooni H (1990) Contact instability of the direct drive robot when constrained by a rigid environment. IEEE Trans Automat Contr 35:710-714.

Kuo PL, Lee DL, Jindrich DL, Dennerlein JT (2006) Finger joint coordination during tapping. J Biomech 39:2934-2942.

Kurtzer I, Herter TM, Scott SH (2005) Random change in cortical load representation suggests distinct control of posture and movement. Nat Neurosci 8:498-504.

Lackner JR, DiZio P (2005) Motor control and learning in altered dynamic environments. Curr Opin Neurobiol 15:653-659.

Lacquaniti F, Maioli C (1987) Anticipatory and reflex coactivation of antagonist muscles in catching. Brain Res 406:373-378.

Lacquaniti F, Maioli C (1989) The role of preparation in tuning anticipatory and reflex responses during catching. J Neurosci 9:134-148.

Lacquaniti F, Maioli C (1994) Independent control of limb position and contact forces in cat posture. J Neurophysiol 72:1476-1495.

Lacquaniti F, Borghese NA, Carrozzo M (1992) Internal models of limb geometry in the control of hand compliance. J Neurosci 12:1750-1762.

Mussa-Ivaldi FA, Bizzi E (2000) Motor learning through the combination of primitives. Philos Trans R Soc Lond B Biol Sci 355:1755-1769.

Ostry D, Feldman A (2003) A critical evaluation of the force control hypothesis in motor control. Exp Brain Res 153:275-288.

Penfield W, Boldrey E (1937) Somatic motor and sensory representation in the cerebral cortex of man as studied by electrical stimulation. Brain 60:389-443.

Santos VJ, Valero-Cuevas FJ (2006) Reported anatomical variability naturally leads to multimodal distributions of Denavit-Hartenberg parameters for the human thumb. IEEE Trans Biomed Eng 53:155-163.

Schieber MH, Hibbard LS (1993) How somatotopic is the motor cortex hand area? Science 261:489-492.

Scott SH (2004) Optimal feedback control and the neural basis of volitional motor control. Nat Rev Neurosci 5:534-546.

Shadmehr R, Mussa-Ivaldi FA (1994) Adaptive representation of dynamics during learning of a motor task. J Neurosci 14:3208-3224.

Sober SJ, Sabes PN (2005) Flexible strategies for sensory integration during motor planning. Nat Neurosci 8:490-497. 
Todorov E (2000) Direct cortical control of muscle activation in voluntary arm movements: a model. Nat Neurosci 3:391-398.

Valero-Cuevas FJ (2000) Predictive modulation of muscle coordination pattern magnitude scales fingertip force magnitude over the voluntary range. J Neurophysiol 83:1469-1479.

Valero-Cuevas FJ (2005) An integrative approach to the biomechanical function and neuromuscular control of the fingers. J Biomech 38:673-684.

Valero-Cuevas FJ, Hentz VR (2002) Releasing the A3 pulley and leaving flexor superficialis intact increases pinch force following the Zancolli lasso procedures to prevent claw deformity in the intrinsic palsied finger. J Orthop Res 20:902-909.

Valero-Cuevas FJ, Zajac FE, Burgar CG (1998) Large index-fingertip forces are produced by subject-independent patterns of muscle excitation. J Biomech 31:693-703.
Valero-Cuevas FJ, Yi JW, Brown D, McNamara III RV, Paul C, Lipson H (2007) The tendon network of the fingers performs anatomical computation at a macroscopic scale. IEEE Trans Biomed Eng 54:1161-1166.

Venkadesan M, Guckenheimer J, Valero-Cuevas FJ (2007) Manipulating the edge of instability. J Biomech 40:1653-1661.

Whitney D (1976) Force feedback control of manipulator fine motions. Productivity 687-693.

Whitney D (1987) Historical perspective and state of the art in robot force control. International J Robotics Res 6:3.

Wolpert DM, Ghahramani Z, Jordan MI (1995) An internal model for sensorimotor integration. Science 269:1880-1882.

Zajac FE (1989) Muscle and tendon: properties, models, scaling, and application to biomechanics and motor control. Crit Rev Biomed Eng 17:359411. 